\title{
Rejection of fixed direction disturbances in multivariable electromechanical motion systems
}

\author{
Matthijs Boerlage* Rick Middleton** Maarten Steinbuch* \\ * Technische Universiteit Eindhoven, Eindhoven, $5600 \mathrm{MB}$ \\ The Netherlands (Tel: +31-40-2472072; e-mail: m.l.g.boerlage@tue.nl). \\ ** Hamilton Institute, The National University of Ireland Maynooth, \\ Co. Kildare, Ireland (e-mail: richard.middleton@nuim.ie)
}

\begin{abstract}
This work discusses rejection of disturbances with known directions in multivariable motion systems. It is shown how frequency domain tradeoffs in multivariable control motivate a design based on disturbance directions. A multivariable design method is presented to design centralized controllers that reject disturbances only in relevant directions. A model of an industrial motion system is used to demonstrate the theory. It is shown how the proposed design method resembles the solution of a competing $\mathcal{H}_{\infty}$ design and offers the ability to interpret $\mathcal{H}_{\infty}$ centralized control solutions, and understand the tradeoffs inherent in such a design.
\end{abstract}

\section{INTRODUCTION}

Multivariable control design problems pose complexity issues as gain, phase and directions in different closed loop transfer functions are strongly interrelated. Therefore, interpretation and development of multivariable control strategies is a challenging problem. In many cases, the structure of the plant can be exploited to reduce this complexity. In Freudenberg [1996], Freudenberg [1999] it is discussed how MIMO control problems can reduce to SIMO or MISO control problems in certain frequency regions or as an effect of certain bandwidth limitations. In Hovd [1997] a class of plants is studied where the input and output directions do not vary with frequency, hence decoupling methods can be applied to reduce the MIMO control problem to a set of SISO control problems. However, in some cases, disturbance have specific directional structure that can give rise to full complexity MIMO control issues, even when plant dynamics are decoupled. This was recognized by some authors, Freudenberg [1988], Maciejowski [1989][page. 85] but there has been little discussion on how this may be exploited in multivariable control design.

In many practical applications, the directions of disturbances are constant (fixed) for all frequencies. Typical examples are disturbances that originate from causes that have a fixed location, e.g., floor vibrations, pumps, fans. Also, the control system architecture can give rise to fixed direction disturbances, see, e.g., Skogestad [1997]. In Boerlage [2007b] a method is discussed to identify the direction and cause of such disturbances. A design that exploits this information for a TITO control system was shown in Boerlage [2007a]. It is of great interest to investigate if the structure of fixed direction disturbances allows the development of insightful MIMO design strategies.

In this work, it is shown that exploiting directions of disturbances is motivated from design limitations induced by integral relations such as the Bode sensitivity integral. Using the structure of a class of electromechanical motion systems, a method is proposed to design controllers that reject fixed direction disturbances only in relevant directions. This method allows frequency wise tradeoffs to be made in each disturbance direction independently, leading to transparent design of a centralized MIMO controller. The method is demonstrated on a design problem of an industrial high performance positioning system. A competing controller is designed using $\mathcal{H}_{\infty}$ synthesis. It is shown that the proposed design method can be used to reverse engineer, hence interpret, the MIMO $\mathcal{H}_{\infty}$ controller.

The paper is organized as follows. The next section shows how design limitations motivate the study of disturbance directions in multivariable control design. Section 3 discusses structural properties of electromechanical motion systems that can be exploited in multivariable control design. The proposed design method to reject disturbances in fixed directions is presented in Section 4. The theory is illustrated by application to an industrial control design problem in Section 5. The last section closes with conclusions.

\section{DESIGN LIMITATIONS}

A feedback control architecture is considered as depicted in Fig. 1. Herein, $d_{i}, d_{o}, n$, denote the input disturbance,

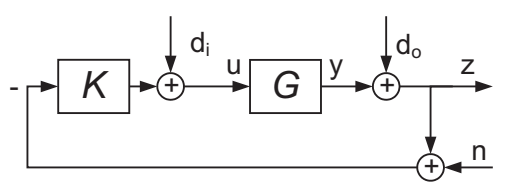

Fig. 1. Control system architecture.

output disturbance and sensor noise respectively. Furthermore, we define the performance variable $z$, plant input $u$ and the plant output $y$. It follows that, 


$$
z=G S_{o} d_{i}+S_{o} d_{o}-T_{o} n
$$

where $S_{o}=(I+G K)^{-1}$ is the output sensitivity function and $T_{o}=G K S_{o}$ the output complementary sensitivity function. Both are algebraically related as $S_{o}+T_{o}=I$. The objective is to keep $z$ as small as possible in presence of $d_{i}, d_{o}$ and $n$. Say that only a disturbance $d_{o}$ with direction $u_{d}$ is present at the output of the plant,

$$
d_{o}(s)=u_{d} v_{d}(s)
$$

And at a single frequency $p$, the singular value decomposition of $S_{o}(p)$ is $S_{o}(p)=V_{S o} \Sigma_{S o} U_{S o}^{H}$. Then, $z(p)$ is small if the last column of $U_{S o}$ is aligned with $u_{d}$, so that $v_{d}(p)$ is amplified with the smallest singular value of $S_{o}$. Apart from alignment, high gain feedback helps to reject disturbances in multivariable systems. However, as analytic design limitations hold, use of high gain feedback for all frequencies and in all directions is restricted in certain situations, Freudenberg [1988].

Consider a stable, non-minimum phase, $n \times n$ multivariable output sensitivity function, $S_{o}(s)$, resulting from a feedback system with stable open loop with relative degree more than two. Then, by pre and post multiplication with vectors $v, u \in \mathbb{C}^{n}, v^{H} u \neq 0$ the following scalar transfer function is obtained,

$$
S_{v u}(s)=v^{H} S_{o}(s) u \text {. }
$$

The response to a disturbance entering the system in a direction spanned by $u$ is given by $S_{o}(s) u$. Then, $v^{H} S_{o}(s) u$ is the component of that disturbance that appears in the output direction spanned by $v$.

Theorem 1. From Freudenberg [1985]. For $S_{v y}$ and $u, v$ defined above, the following integral relation applies,

$$
\int_{0}^{\infty} \log \left|S_{v u}(j \omega)\right| d \omega=\frac{\pi}{2} \log \left|v^{H} u\right|+\pi \sum_{i=1}^{N_{z}} z_{i}
$$

with $z_{i}, i=\left\{1, \ldots, N_{z}\right\}$ the CRHP zeros of $S_{v u}$.

Proof 1. As $S_{o}$ is stable, the scalar transfer function $S_{v u}$ is stable, and has at most the relative degree of $S_{o}(s)$. So that at high frequencies, it holds that,

$$
\lim _{s \rightarrow \infty} v^{H} S_{o}(j \omega) u=\left|v^{H} u\right|
$$

Even if $S_{o}$ is minimum phase, $S_{v u}$ can become nonminimum phase, hence introduce zeros in the closed right half plane. Hence, scalar integral relations apply, Freudenberg [1985].

This implies that an area where $\left|S_{v u}(j \omega)\right|$ is small, must be balanced by an equal area where $\left|S_{v u}(j \omega)\right|$ is large.

In practical applications, the bandwidth of the feedback system is limited, e.g., by plant uncertainty at high frequencies. Hence, the frequency range with large $\left|S_{v u}(j \omega)\right|$ is constrained. Then, Theorem 1 , shows that rejection of disturbances at other frequencies, where $\left|S_{v u}(j \omega)\right|$ must be small, has to be compromised. This may imply performance limiting tradeoffs. Specific choices of $v, u$ illustrate how these frequency wise tradeoffs dictate multivariable control design.

Corollary 2. When $v, u$ are chosen to be elementary vectors $e_{i}, S_{v u}(s)=S_{o, i i}(s)$ equals the $i^{t h}$ diagonal element of the sensitivity transfer function matrix. As $e_{i}^{T} e_{i}=1$, Theorem 1 reduces to the bode integral relation for scalar systems. Hence, for each scalar loop in a multivariable system, the scalar bode integral relation must be satisfied, even if a centralized linear time invariant controller is applied.

Corollary 3. To study non-diagonal terms of the sensitivity transfer function matrix, $v, u$ can be chosen to approach orthogonal elementary vectors, $e_{i}^{H} e_{j}=\epsilon, 0<\epsilon \ll 1$, $S_{v u}(s)=e_{i}^{T} S_{o}(s) e_{j}$. Then,

$$
\lim _{\epsilon \rightarrow 0} \log \left(\left|e_{i}^{H} e_{j}\right|\right)=-\infty .
$$

This shows that a single non-diagonal term of a sensitivity function matrix can be made arbitrarily small, even in cases where the open loop is non-diagonal. However, as will be shown later, it may be desired to increase non-diagonal terms of a sensitivity function for the sake of disturbance rejection.

Corollary 4. By choosing $u_{i}=v_{j} \in \mathbb{R}^{n}, v_{i}^{T} v_{j}=0$ and $M=\operatorname{Span}\left\{v_{1}, v_{2}, \ldots, v_{n}\right\}$ an orthogonal transformation $S_{o, M}(s)=M^{T} S_{o}(s) M$ can be made. As this is a nonsingular input output transformation, $S_{o, M}$ is minimum phase if $S_{o}$ is minimum phase. Then on each new base Theorem 1 holds with $N_{z}=0$.

If the direction of a disturbance is constant for all frequencies, and the performance is defined as a constant linear combination of $z$, see (1), rejection of that disturbance at one frequency implies that the sensitivity function has to be increased at other frequencies in that same direction. Hence, as specifications become tighter, it is crucial for design to reject disturbances only at frequencies and in directions that are relevant. This may imply that centralized controllers (with non-diagonal terms) are to be designed, even in cases where the plant is decoupled.

\section{ELECTROMECHANICAL MOTION SYSTEMS}

We first show that the multivariable plants we consider have a specific structure. Here, we focus on the control of linear time invariant electromechanical motion systems that have the same number of actuators and sensors as rigid body modes. Typical applications are high performance positioning stages used in semiconductor manufacturing, electron microscopy or component placement machines. The dynamics of such systems are often dominated by the mechanics, which are therefore constructed to be light and stiff, so that resonance modes due to flexible dynamics appear only at high frequencies. Typically, flexible dynamics have low internal damping, so that it is justified to assume proportional damping. Then, the following model can be used to describe the dynamics of the plant, Gawronski [2004],

$$
G_{m}(s)=\sum_{i=1}^{N_{r b}} \frac{c_{i} b_{i}^{T}}{s^{2}}+\sum_{i=N_{r b}+1}^{N} \frac{c_{i} b_{i}^{T}}{s^{2}+2 \zeta_{i} \omega_{i} s+\omega_{i}^{2}}
$$

Herein, $N_{r b}$ denotes the number of rigid body modes. The parameters $\zeta_{i}, \omega_{i}$ are the relative damping and resonance frequency of the flexible modes. The vectors $c_{i}, b_{i}$ span the directions of the $i^{\text {th }}$ mode shapes and are constant for all frequencies. The resonance frequencies $\omega_{i}$ are high, hence the plant can be approximately decoupled using static input (and/or output) transformations, $T_{u}, T_{y}$ respectively so that, 


$$
\begin{aligned}
G_{y u}(s) & =T_{y} G_{m}(s) T_{u} \\
& =G(s)+G_{\text {flex }}(s), \quad G(s)=\frac{1}{m s^{2}} I
\end{aligned}
$$

where $m \in \mathbb{R}^{1}$ and $G_{\text {flex }}(s)$ contains the flexible dynamics of the plant and is often non-diagonal. In many applications, the frequencies and damping of the resonance modes changes in the life cycle of the plant and is sensitive to changes in operating point. Hence, inversion of these dynamics leads to robustness problems. The objective is to control the rigid body behavior of the plant with high fidelity. The influence of the flexible dynamics can then modeled as a multiplicative perturbation at the output of the plant $G(s)$,

$$
G_{y u}(s)=(I+E(s)) G(s)
$$

with $E(s)=G_{f l e x}(s) G^{-1}(s)$, see also Fig. 2. With a

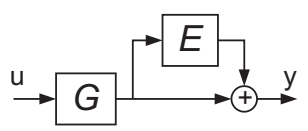

Fig. 2. Multiplicative perturbation at the output of the plant to model influence of flexible dynamics.

multivariable controller $K(s)$, the plant $G(s)=\frac{1}{m s^{2}} I$, and stable sensitivity function $S_{o}=(I+G K)^{-1}$, the closed loop system is stable when $\rho\left(E(j \omega) T_{o}(j \omega)\right)<1, \forall \omega$, Grosdidier [1986]. A sufficient condition for this is,

$$
\bar{\sigma}\left(T_{o}(j \omega)\right)<\bar{\sigma}\left(E(j \omega)^{-1}, \forall \omega .\right.
$$

As $E(j \omega)$ is large at high frequencies, this shows that the flexible dynamics limit the bandwidth of the closed loop system. Hence, one is forced to make frequency wise tradeoffs if disturbances are to be rejected in electromechanical motion systems.

\section{DESIGN FOR FIXED DIRECTION DISTURBANCES}

A method is presented to design a controller that rejects disturbances with a fixed direction for the class of electromechanical motion systems discussed earlier. We focus on the rejection of output disturbances that can be represented by the following model,

$$
d_{o}(s)=U V_{d}(s) \text {. }
$$

Herein, $V_{s}(s)$ is a square diagonal transfer function matrix and $U$ is a non-singular matrix with constant elements for all frequencies. In the following, we restrict ourselves to the case where $U$ is an orthogonal matrix. The theory is not limited to this, a few remarks on more general choices of $U$ will be given at the end of this section.

Originally, the plant is defined in the control coordinates. A coordinate transformation $y_{d}=U^{T} y$ so that, $U^{T} d_{o}(s)=V_{d}(s)$, can be used to express the variables in disturbance coordinates. This is equivalent to choosing a controller $K(s)=U K_{d}(s) U^{T}$, with $U \in \mathbb{R}^{n \times n}, U^{T} U=I$ and $K_{d}(s)$ a diagonal matrix with scalar transfer functions. For the class of plants considered here holds that, $G(s)=g(s) I$. Hence, the transfer functions at the right hand side of (1), become,

$$
\begin{aligned}
S_{o} & =U S_{o}^{d}(s) U^{T} \\
T_{o} & =U g(s) K_{d}(s) S_{o}^{d}(s) U^{T} \\
S_{o} G & =U S_{o}^{d}(s) g(s) U^{T}
\end{aligned}
$$

where $S_{o}^{d}(s)=\left(I+g(s) K_{d}(s)\right)^{-1}$, is the (diagonal) sensitivity function in disturbance coordinates. Therefore, all relevant closed loop transfer functions are decoupled in the disturbance coordinates. This means that a disturbance with direction aligned to a column of $U^{T}$, is only affected by a single controller on the diagonal of $K_{d}(s)$. Also, by Corollary 4, the frequency domain tradeoff, implied by rejecting such a disturbance, only manifests itself in the diagonal element of $S_{o}^{d}(s)$ corresponding to the diagonal element of $K_{d}(s)$. At the same time, a single element of $K_{d}(s)$ can change more elements of $S_{o}(s)$, which is in control coordinates.

Considering the influence of the flexible dynamics, modeled as a multiplicative perturbation $E(s)$, Fig. 2, the transformation implies that the multiplicative perturbation in disturbance coordinates equals, $E_{d}(s)=U^{T} E(s) U$. As $U$ is orthogonal, $\bar{\sigma}\left(E_{d}\right)=\bar{\sigma}(E)$ and the same upper bound as shown in (10) has to be satisfied for all elements of the diagonal complementary sensitivity function in disturbance coordinates, $T_{o}^{d}(s)$.

The same coordinate transformation can be performed with any invertible matrix $U$, so that $K(s)=U K_{d}(s) U^{-1}$. However, the consequence is that the bound on $T_{o}^{d}(s)$ induced by $E$, can be conservative if the condition number of $U$ increases as $\bar{\sigma}\left(U E U^{-1}\right) \leq \bar{\sigma}(E) \kappa(U)$, where $\kappa(U)=\bar{\sigma}(U) / \underline{\sigma}(U)$ is the condition number. Likewise, specifications as the peak value of the sensitivity functions are difficult to carry over to disturbance coordinates if $\kappa(U)$ is large.

As the rejection of fixed direction disturbances is studied in coordinates where the disturbances are decoupled, frequency domain tradeoffs are more transparent. Because the controller and all closed loop transfer functions in disturbance coordinates are diagonal, scalar design techniques, such as manual loopshaping see e.g. Steinbuch [1998], can be facilitated. The resulting controller in control coordinates, $K(s)$, will in general be non-diagonal as the input directions of the sensitivity function in control coordinates $S_{o}(s)$ will be aligned to the disturbance directions (that can be non-canonical). The following example will show how the proposed method can be used in a practical design problem.

\section{APPLICATION EXAMPLE}

As a demonstration of the theory, a model of a high precision positioning stage is studied. A detailed description of the plant can be found in van de Wal [2002]. The objective is to regulate the position of this stage in presence of environmental disturbances, e.g. floor vibrations, machine oscillations, pumps, etc. As the location of the origin of these disturbance is fixed, the directions of these disturbances are fixed. Identification of the direction and the origin of such disturbances is discussed in Boerlage [2007b]. The stage has six degrees of freedom, here only the cartesian axis $x, y, z$ (and interaction in between) are studied. It is a 
design requirement that the plant is expressed in these cartesian coordinates. The Bode magnitude diagram of the $G_{y u}(s)$ is shown in Fig. 3. Note that the index of the frequency axis is removed for commercially sensitive reasons, frequencies that are discussed in this work are indicated by numbers. Following the approach in Section 3 , the plant to be controlled equals,

$$
G(s)=\frac{1}{m s^{2}} I
$$

where $m \in \mathbb{R}^{1}$, Fig. 4 . Flexible dynamics $G_{\text {flex }}(s)$, Fig. 4 , limit the achievable bandwidth as the inverse of the maximum singular value of $E(s)$ upper bounds the allowable complementary sensitivity, see Fig. 8. For robustness purposes it is required that $\bar{\sigma}\left(S_{o}\right)<6 \mathrm{~dB}$. The rejection of three harmonics in orthogonal directions is studied. Each harmonic is described by a scalar model $V_{d i}(s), i=$ $\{1,2,3\}$, representing the harmonic at frequency $1,2,3[-]$ respectively. The disturbance at the output of the plant then equals,

$$
d_{o}(s)=U V_{d}(s)
$$

where $V_{d}(s)=\operatorname{diag}\left\{V_{d 1}(s), V_{d 2}(s), V_{d 3}(s)\right\}$ and $U$ is a constant orthogonal matrix,

$$
U=\frac{1}{3}\left[\begin{array}{rrr}
2 & -2 & 1 \\
1 & 2 & 2 \\
2 & 1 & -2
\end{array}\right]
$$

which columns span the directions of the disturbances. In the next subsections, three design approaches are considered that are designed to have the same level of rejection of these disturbances.
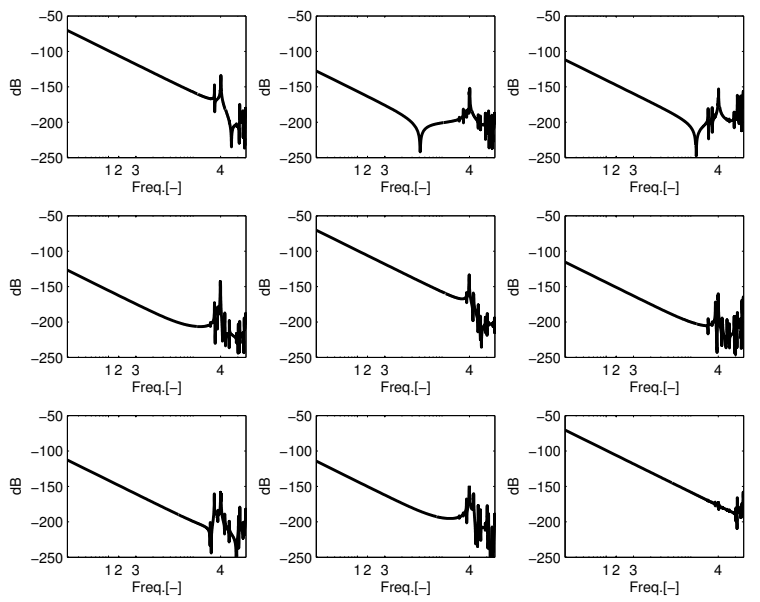

Fig. 3. Bode magnitude diagram of the high precision positioning stage.

\subsection{Multiloop SISO design in control coordinates}

This case illustrates how a conventional decentralized control approach handles rejection of disturbances in a multivariable system. As the plant is decoupled, a typical approach is to design a multiloop SISO controller (lead lag, second order lowpass) and extend each SISO controller with a collection of inverted notches (bandpass filters) tuned at the components of the disturbances at the output of the plant, Fig. 5. As in this approach, the directions
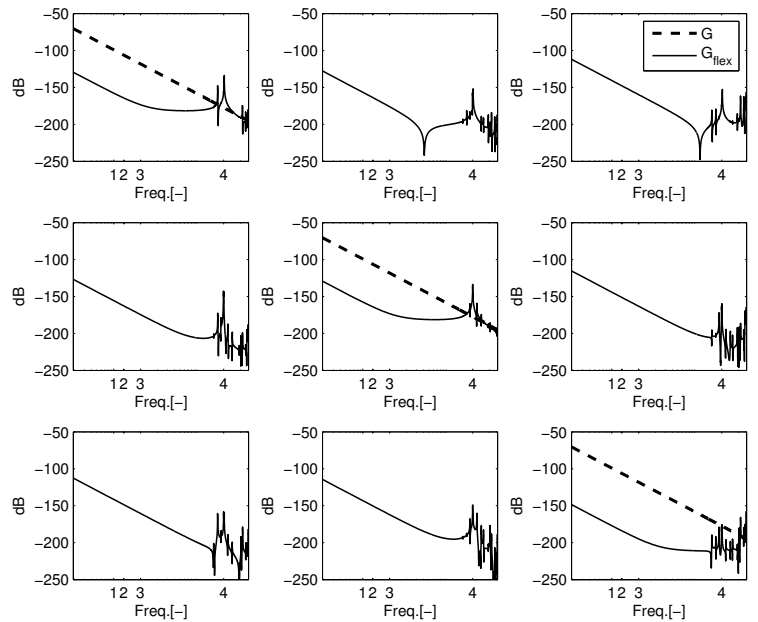

Fig. 4. Bode magnitude diagram of $G$ and $G_{f l e x}$.
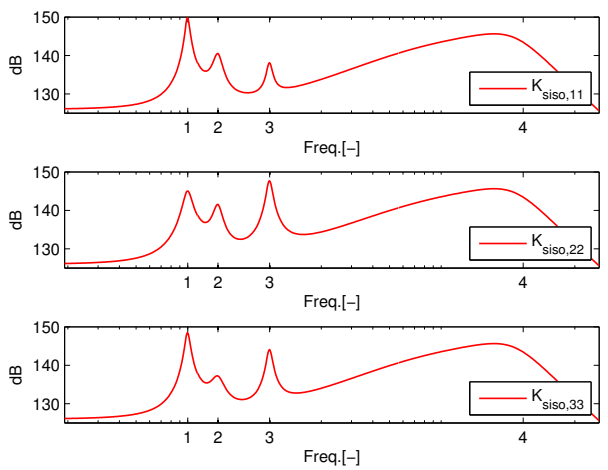

Fig. 5. Bode magnitude diagram of the diagonal terms of the multiloop SISO controller (in control coordinates).

of the disturbances are not taken into account explicitly, the disturbances at each $j^{\text {th }}$ output of the plant are then modeled as,

$$
d_{o, j}(s)=\sum_{i=1}^{3} U_{j, i} V_{d i}(s)
$$

where $U_{j, i}$ denotes the $j, i^{\text {th }}$ element of $U$. It turns out to be very difficult to reject these disturbances while at the same time satisfing the other design requirements, $\bar{\sigma}\left(S_{o}\right)<6[d B], \bar{\sigma}\left(T_{o}\right)<\bar{\sigma}(E)^{-1}$. Rejection of the disturbances is only possible if either the sensitivity peak increases, or the complementary sensitivity function crosses the bound induced by the flexible dynamics, Fig. 8. This typical limitation can be explained as in Fig. 9 it is visible that the sensitivity function is reduced in directions that are not relevant for this design case. Hence the sensitivity function should be large at more frequencies then necessary. This design approach does not result in a satisfactory solution for this problem.

\subsection{Design in disturbance coordinates}

A $3 \times 3$ MIMO controller is designed in disturbance coordinates, following the approach in Section 4. Each element of 

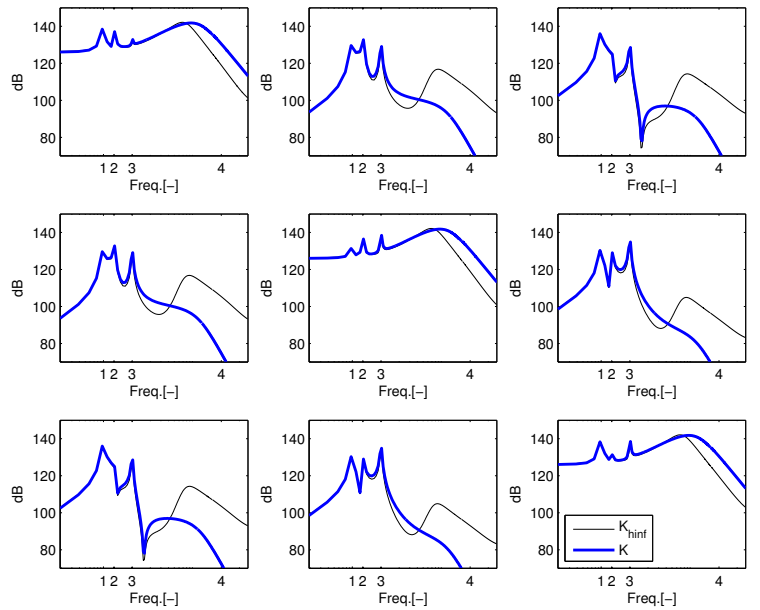

Fig. 6. Bode magnitude diagram of the controller in control coordinates, $K_{\text {hinf }}$ and $K$.
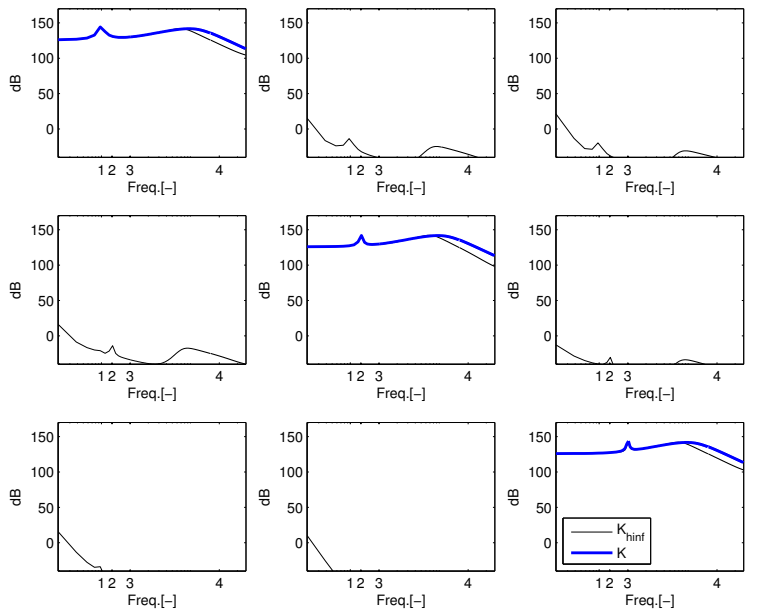

Fig. 7. Bode magnitude diagram of the controller in disturbance coordinates, $K_{\text {hinf }}$ and $K$.

the (diagonal) controller in disturbance coordinates affects a single (diagonal) term of $S_{o}^{d}(s)$, that has to be small at frequencies where $V_{d i}(s)$ is large. A lead lag controller with second order low pass filter is designed for each loop. The harmonic disturbance is rejected using a single inverted notch (band pass) filter, tuned to a single frequency, $1,2,3[-]$, for each loop respectively. The bandwidth is limited by $\bar{\sigma}(E)$, Fig. 8, which was shown to be invariant under orthogonal coordinate transformation. The bode magnitude diagram of the controller, $K^{d}(s)$, is depicted in Fig. 7. The controller in control coordinates equals $U K^{d}(s) U^{T}$ and is shown in Fig. 6. In Fig. 9 it is visible that each principal gain is small at only one frequency of the disturbance. Hence, the sensitivity function is small only in the directions that are relevant for this disturbance model.

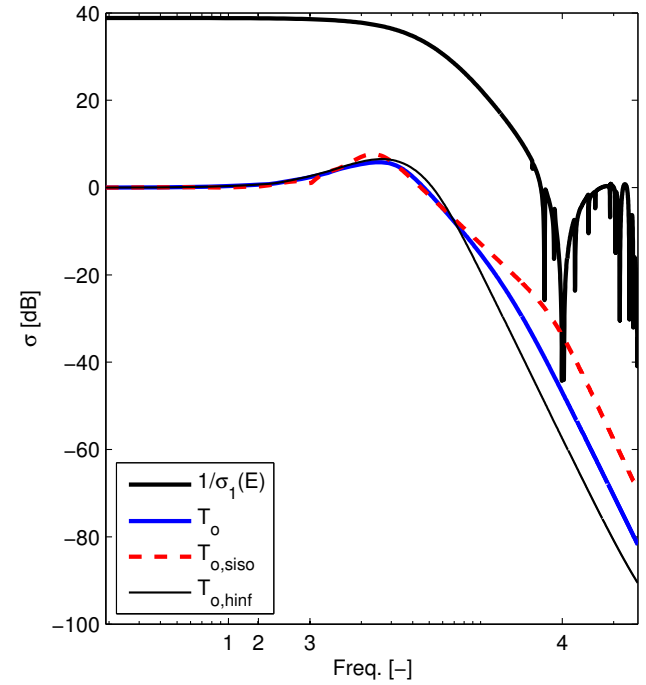

Fig. 8. Maximum singular value of complementary sensitivity for $\mathcal{H}_{\infty}$ design, SISO design and design in disturbance coordinates compared to the bound induced by $E(j \omega)$.
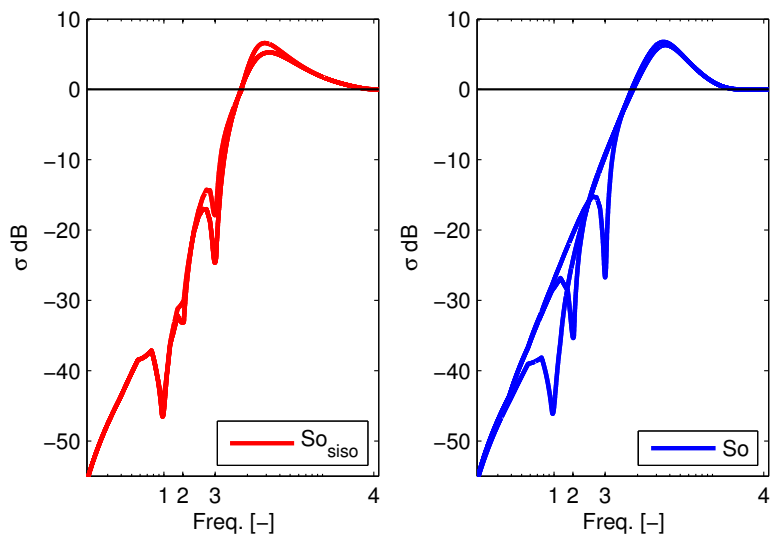

Fig. 9. Principal gains of the output sensitivity function. Multiloop SISO design (left) and design in disturbance coordinates (right).

\section{$5.3 \mathcal{H}_{\infty}$ design in control coordinates}

The objective is to design a $3 \times 3$ MIMO controller using $\mathcal{H}_{\infty}$-synthesis, that takes into account the directions of the disturbances. The design is formulated in control coordinates. Synthesis of the $\mathcal{H}_{\infty}$ controller is based on the following mixed sensitivity formulation,

$$
\underset{\text { min }}{\operatorname{mib.} K_{\text {hinf }}}\left\|\begin{array}{c}
W_{s} S_{o} V \\
W_{k s} K S_{o} V
\end{array}\right\|_{\infty} .
$$

Herein, $W_{s}(s)$ and $W_{k s}(s)$ are chosen diagonal, see Fig. 10 , see van de Wal [2002] for guidelines. The weight $V(s)$ models the disturbances at the output of the plant and equals,

$$
V(s)=U V_{d}(s)
$$

where $V_{d}(s)$ is the diagonal transfer function matrix from (14). Note that as $V(s)$ is non-diagonal, the generalized plant becomes coupled, and there is no reason 


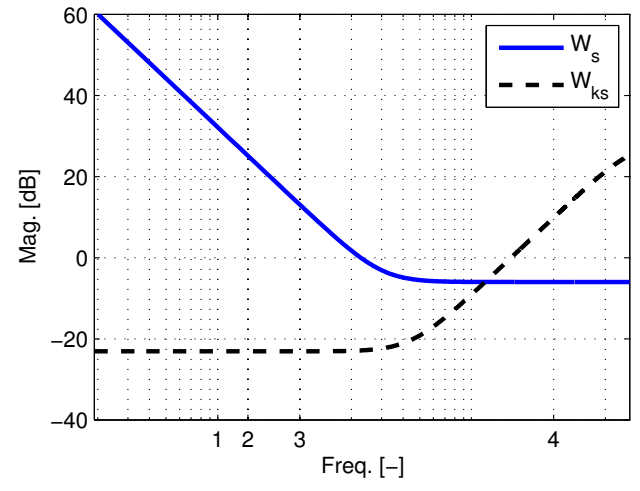

Fig. 10. Weighting filters used in $\mathcal{H}_{\infty}$ design.

why the resulting $\mathcal{H}_{\infty}$ controller should be diagonal. The resulting $\mathcal{H}_{\infty}$ controller indeed turns out to have large non-diagonal terms, see Fig. 6. However, if the same $\mathcal{H}_{\infty}$ controller is transformed to disturbance coordinates using, $U^{T} K_{\text {hinf }}(s) U$, it is visible, Fig. 7 , that $\mathcal{H}_{\infty}$ synthesis comes up with the same solution as our manual design in disturbance coordinates, at least in the frequency region of the disturbances. The $\mathcal{H}_{\infty}$ controller has a different roll off at higher frequencies. This leads to better roll off of the singular values of $T_{o}$, but seems to result in slightly larger cross-terms of the complementary sensitivity function at these frequencies.

\subsection{Discussion}

Only the $\mathcal{H}_{\infty}$ control and the manual control design in disturbance coordinates are shown to provide feasible solutions to this disturbance rejection problem. This is explained as these approaches allow the designer to exploit specific directional properties of disturbances. Hence, the sensitivity function is reduced only in those directions that are relevant. A conventional multiloop design approach was shown to be overly conservative and hence dictates frequency domain tradeoffs that made it impossible to satisfy the design requirements. For comparison, each SISO controller in the multiloop approach was designed using $\mathcal{H}_{\infty}$-synthesis (results not shown here). As these designs still have to face the same frequency domain tradeoffs, no feasible control design could be obtained.

The spectral radius equals $\rho\left(E(j \omega) T_{o}(\omega)\right)=0.70$ for the design in disturbances coordinates, achieved at frequency $4[-]$, see also Fig. 8. And $\rho=0.19, \rho=3.13$ for the $\mathcal{H}_{\infty}$ controller and multiloop respectively. This again shows that the multiloop design is not able to satisfy the design requirements. The order of the $\mathcal{H}_{\infty}$ controller is 42 . The SISO design must duplicate notches in each loop and has order 27. The order of the controller designed in disturbance coordinates is 15 . Aside from this advantage, the design in disturbance coordinates is more transparent as a controller can be designed for each harmonic disturbance independently. Also, this approach resulted in approximately the same controller as the $\mathcal{H}_{\infty}$ design. A possible, more refined, design strategy is to choose different bandwidths for the controllers in each disturbance direction so that robustness margins or roll off can be increased in those directions.
Although the manual design in disturbance coordinates is only applicable for a limited class of plants, it does illustrate how rejection of fixed direction disturbances can be achieved in a transparant way. It is of great practical value to be able to interpret MIMO controllers that result from $\mathcal{H}_{\infty}$ synthesis in this class of problems.

\section{CONCLUSIONS}

It is discussed and illustrated that rejection of disturbances in only the relevant directions is important in design problems with tight specifications. This is motivated by a scalar integral relation, describing the disturbance response per direction. Exploiting the structure of a class of electromechanical systems, a coordinate transformation can be applied that allows to transparently, even manually, design centralized multivariable controllers that reject disturbances only in the relevant directions. If the same problem is approached with $\mathcal{H}_{\infty}$ design, and the resulting MIMO $\mathcal{H}_{\infty}$ controller is transformed to the same coordinates, the $\mathcal{H}_{\infty}$ controller is shown to do exactly the same.

\section{REFERENCES}

M. Boerlage, B. de Jager, M. Steinbuch Multivariable control design for fixed direction disturbances. American Control Conference, New York, pages 4649-4654, 2007.

M. Boerlage, B. de Jager, M. Steinbuch Blind identification of fixed direction disturbances. Multi-conference on Systems and Control, Singapore, in printing, 2007.

J. Freudenberg and D. Looze. Right Half Plane Poles and Zeros and Design Tradeoffs in Feedback Systems. IEEE Transactions on Automatic Control, volume 30, number 6, pages 555-565, 1985.

J. Freudenberg and D. Looze. Frequency Domain Properties of Scalar and Multivariable Feedback Systems. Lecture Notes in Control and Information Sciences, Springer-Verlag, 1988.

J. Freudenberg and R. Middleton Design Rules for Multivariable Feedback Systems. Conference on Decision and Control, Kobe, pages 1980-1985, 1996.

J. Freudenberg, R. Middleton Properties of single input, two output feedback systems. International Journal of Control, volume 72, issue 16, pages 1446 - 1465, 1999.

W. Gawronski. Advanced Structral Dynamics and Active Control of Structures. Springer-Verlag, 2004.

P. Grosdidier and M. Morari. Interaction measures for systems under decentralized control. Automatica, volume 22, issue 3, pages 309-319, 1986.

M. Hovd, R. Braatz and S. Skogestad SVD Controllers for $H_{2}-, H_{\infty}-$ and $\mu$-optimal Control. Automatica, volume 33, number 3, pages 433-439, 1997.

J. Maciejowski Multivariable feedback design. AddisonWesley, 1989.

S. Skogestad and M. Morari. Effect of Disturbance Directions on Closed-Loop Performance. Industrial \& Engineering Chemistry Research, volume 26, number 1, pp.2029-2035, 1987.

M. Steinbuch and M. Norg. Advanced motion control: an industrial perspective. European Journal of Control, pp.278-293, 1998.

M. van de Wal, G. van Baars, F. Sperling and O. Bosgra Multivariable $\mathcal{H}_{\infty} / \mu$ feedback control design for highprecision wafer stage motion. Control Engineering Practice, volume 10, issue 7, pages 739-755, 2002. 